Review Article

\title{
Extremal problems for graphical function-indices and $f$-weighted adjacency matrix
}

\author{
Xueliang $\mathrm{Li}^{\dagger}$, Danni Peng \\ Center for Combinatorics and LPMC, Nankai University, Tianjin 300071, China
}

(Received: 11 January 2022. Received in revised form: 19 January 2022. Accepted: 28 January 2022. Published online: 5 February 2022.)

(C) 2022 the authors. This is an open access article under the CC BY (International 4.0) license (www.creativecommons.org/licenses/by/4.0/).

\begin{abstract}
Let $f(x, y)(f(x))$ be a symmetric real function (real function) and $G=(V, E)$ be a graph. Denote by $d_{i}$ the degree of a vertex $i$ in $G$. The graphical function-index $T I_{f}(G)\left(H_{f}(G)\right)$ of $G$ with edge-weight (vertex-weight) function $f(x, y)(f(x))$ is defined as $T I_{f}(G)=\sum_{u v \in E} f\left(d_{u}, d_{v}\right)\left(H_{f}(G)=\sum_{u \in V} f\left(d_{u}\right)\right)$. We can also get a weighted adjacency matrix from the edge-weighted graph, i.e., $A_{f}(G)=\left(a_{i j}^{f}\right)$ where $a_{i j}^{f}=f\left(d_{i}, d_{j}\right)$ if vertices $i$ and $j$ are adjacent in $G$, and 0 otherwise. This matrix is simply referred to as the $f$-weighted adjacency matrix. One can see that the concepts of graphical function-indices and $f$-weighted adjacency matrix can cover all the degree-based graphical indices and degree-based adjacency matrices of graphs, such as the Zagreb indices, Randić index, ABC-index, etc., and the Randić matrix, ABC-matrix, GA-matrix, etc. So, for the graphical function-indices $T I_{f}(G)$ and $H_{f}(G)$ and the $f$-weighted adjacency matrix $A_{f}(G)$ of a graph $G$, one can think about finding unified ways to study the extremal problems and spectral problems. This survey is intended to sum up the results done so far on these problems.
\end{abstract}

Keywords: graphical function-index; edge (vertex)-weight function; $f$-weighted adjacency matrix; extremal problems.

2020 Mathematics Subject Classification: 05C09, 05C92.

\section{Notation and terminology}

First, we give the necessary notation and terminology which will be used throughout this paper. We only consider simple and finite graphs in this paper. For notation and terminology not defined here, we refer the reader to $[4,45]$. We use $V(G)$ and $E(G)$ to denote the vertex-set and edge-set of a graph $G$, respectively. A graph $G$ is called $k$-regular if the degree $d(v)=k$ for every $v \in V(G)$. Denote the degree of a vertex $v$ in $G$ also by $d_{v}$. An $(n, m)$-graph is a graph $G=(V(G), E(G))$, where $m=|E(G)|$ and $n=|V(G)|$. Let $G(n, m)$ represent the collection of all $(n, m)$-graphs, $G(n)$ represent the collection of all graphs with $n$ vertices but without isolated vertices. We denote by $\Delta$ and $\delta$ the maximum degree and minimum degree of $G$, respectively. A graph $G$ is called almost regular if $\Delta-\delta \leq 1$ and $\mathcal{G}(n, m)$ is the family of all almost regular $(n, m)$-graphs. Note that for an $(n, m)$-graph $G$, if we let $k=\lfloor 2 m / n\rfloor$ and $r=2 m-k n \in\{0,1, \ldots, n-1\}$, then $G$ belongs to $\mathcal{G}(n, m)$ if and only if $G$ has $r$ vertices of degree $k$ and $n-r$ vertices of degree $k+1$.

As usual, let $A(G)=\left(a_{i j}\right)$ denote the adjacency matrix of $G$, where

$$
a_{i j}= \begin{cases}1 & \text { if } i \text { and } j \text { are adjacent in } G, \\ 0 & \text { otherwise }\end{cases}
$$

Then, $A(G)$ is a symmetric $(0,1)$-matrix, and therefore all its eigenvalues are real numbers, say $\mu_{1}, \mu_{2}, \cdots, \mu_{n}$. The spectral radius of $A$ is $\rho(A)=\max _{1 \leq i \leq n}\left|\mu_{i}\right|$.

For $k$ graphs $G_{1}, G_{2}, \ldots, G_{k}$, the union $G_{1} \cup G_{2} \cup \cdots \cup G_{k}$ is the graph with vertex-set $V\left(G_{1}\right) \cup V\left(G_{2}\right) \cup \cdots \cup V\left(G_{k}\right)$ and edge-set $E\left(G_{1}\right) \cup E\left(G_{2}\right) \cup \cdots \cup E\left(G_{k}\right)$. In particular, if $G_{1}=G_{2}=\cdots=G_{k}=G$, we denote $G_{1} \cup G_{2} \cup \cdots \cup G_{k}$ by $k G$. The join $G \vee H$ of two graphs $G$ and $H$ is the graph obtained by joining edges between each vertex of $G$ to all vertices of $H$. We use $C_{n}, P_{n}$ and $S_{n}$ to denote a cycle, a path and a star on $n$ vertices, respectively. $S_{d, n-d}$ represents a double star on $n$ vertices with degrees of the two centers equal to $d$ and $n-d$, respectively.

\section{Background}

Nowadays, chemical graph theory has become a popular and important subject of study. In the mathematics subject classification 2020, "chemical graph theory" is given a subject number 05C92 in "05C Graph theory". Also, "graphical 
indices (Wiener index, Zagreb index, Randić index, etc.)" is given an independent subject number 05C09. The popular book [45] should be considered as one of the pioneer works on chemical graph theory.

There are many chemical indices [42], such as the first Zagreb index $M_{1}=\sum_{u \in V(G)} d_{u}^{2}$ or $\sum_{u v \in E(G)}\left(d_{u}+d_{v}\right)$ [21], the second Zagreb index $M_{2}=\sum_{u v \in E(G)} d_{u} d_{v}$ [21], the Randić index $\chi=\sum_{u v \in E(G)}\left(d_{u} d_{v}\right)^{-1 / 2}$ [40], and the Wiener index $W=\sum_{\{u, v\} \subset V(G)} \operatorname{dist}(u, v)$, where $\operatorname{dist}(u, v)$ is the distance between $u$ and $v$. Usually, these chemical indices are classified into some categories: degree-based, distance-based, subgraph counting-based, eigenvalue-based, mixed, etc. In this survey, we are only concerned with the degree-based indices. We list some of the degree-based indices in Table 1; for more such indices, we refer to $[18,20,33]$. In the past years, a lot of work have been done on these degree-based indices; see $[2,3,7,14,17,21,22,24,25,28,29,34,41,49]$ and the references therein.

Table 1: Some edge-weight functions and related chemical indices.

\begin{tabular}{|c|c|}
\hline$f(x, y)$ & Name \\
\hline$x+y$ & First Zagreb index \\
\hline$x y$ & Second Zagreb index \\
\hline$(x+y)^{2}$ & First hyper-Zagreb index \\
\hline$(x y)^{2}$ & Second hyper-Zagreb index \\
\hline$x^{-3}+y^{-3}$ & Modified first Zagreb index \\
\hline$|x-y|$ & Albertson index \\
\hline$(x / y+y / x) / 2$ & Extended index \\
\hline$(x-y)^{2}$ & Sigma index \\
\hline $1 / \sqrt{x y}$ & Randić index \\
\hline$\sqrt{x y}$ & Reciprocal Randić index \\
\hline $1 / \sqrt{x+y}$ & Sum-connectivity index \\
\hline$\sqrt{x+y}$ & Reciprocal sum-connectivity index \\
\hline $2 /(x+y)$ & Harmonic index \\
\hline$\sqrt{(x+y-2) /(x y)}$ & $\mathrm{ABC}$ index \\
\hline$[x y /(x+y-2)]^{3}$ & Augmented Zagreb index \\
\hline$x^{2}+y^{2}$ & Forgotten index \\
\hline$x^{-2}+y^{-2}$ & Inverse degree \\
\hline $2 \sqrt{x y} /(x+y)$ & Geometric-arithmetic index \\
\hline$(x+y) / 2 \sqrt{x y}$ & Arithmetic-geometric index \\
\hline$x y /(x+y)$ & Inverse sum index \\
\hline$x+y+x y$ & First Gourava index \\
\hline$(x+y) x y$ & Second Gourava index \\
\hline$(x+y+x y)^{2}$ & First hyper-Gourava index \\
\hline$[(x+y) x y]^{2}$ & Second hyper-Gourava index \\
\hline $1 / \sqrt{x+y+x y}$ & Sum-connectivity Gourava index \\
\hline$\sqrt{(x+y) x y}$ & Product-connectivity Gourava index \\
\hline$\sqrt{x^{2}+y^{2}}$ & Sombor index \\
\hline
\end{tabular}


From Table 1, one can see that these chemical indices have the following unified definition. Let $f(x, y)$ be a symmetric real function and $G$ be a graph. The degree-based topological index or chemical index can be defined as

$$
T I_{f}(G)=\sum_{u v \in E(G)} f\left(d_{u}, d_{v}\right)
$$

which was mentioned by Gutman in [19]. This was also called the bond-incident-degree index BID $(G)$ by Vukičević and Durdević in [46]. We also address it as the graphical function-index of a graph with edge-weight function $f(x, y)$. This topological index can also be represented as $T I_{f}(G)=\sum_{(i, j) \in K} m_{i j} f(i, j)$, where $m_{i j}$ is the number of edges between a vertex of degree $i$ and a vertex of degree $j$ and $K=\{(i, j) \in \mathbb{N}: 1 \leq i \leq j \leq n-1\}$. For a family $\mathcal{G}$ of graphs, we call a graph $G$ minimal in $\mathcal{G}$ if $T I_{f}(G)=\min _{H \in \mathcal{G}} T I_{f}(H)$, and maximal in $\mathcal{G}$ if $T I_{f}(G)=\max _{H \in \mathcal{G}} T I_{f}(H)$.

Another graphical function-index introduced by Linial and Rozenman in [36] is defined as follows. Let $f(x)$ be a real function. The graphical function-index $H_{f}(G)$ of $G$ with vertex-weight function $f(x)$ is defined as

$$
H_{f}(G)=\sum_{u \in V(G)} f\left(d_{u}\right)
$$

Notice that by taking the symmetric real function $f(x, y)$ equal to $f(x) / x+f(y) / y$ for some real function $f(x)$, one can deduce that $H_{f}(G)$ is a special case of $T I_{f}(G)$.

One can see that from a graphical index, one gets a number from an edge-weighted or vertex-weighted graph, i.e., each index maps a molecular graph to a single number, which hopefully represents the structure of the graph. We often get the conclusion that for any graphical index $\phi$, it is almost sure (with high probability) that for every graph $G$ there is another graph $G^{\prime}$ such that $\phi\left(G^{\prime}\right)=\phi(G)$ but $G^{\prime}$ is not isomorphic to $G$. This is a sad news for people trying to invent graphical indices that can distinguish among graph structures. Nevertheless, if we use a matrix to represent the structure of a molecular graph with weights separately distributed on its pairs of adjacent vertices, it will completely keep the structural information of the graph, i.e., a matrix keeps much more structural information than an index. This motivates us to introduce the following notion of matrix. Let $f(x, y)$ be a symmetric real function, and denote by $d_{i}$ the degree of a vertex $i$ in $G$. Then a weighted adjacency matrix $A_{f}(G)$ of $G$ is defined in [12] as follows: the $i j$-entry of $A_{f}(G)$ is

$$
A_{f}(G)(i, j)= \begin{cases}f\left(d_{i}, d_{j}\right), & i j \in E(G), \\ 0, & \text { otherwise. }\end{cases}
$$

So, we get a symmetric real matrix, and call it the function-weighted adjacency matrix of $G$, or simply $f$-weighted adjacency matrix, with edge-weight function $f(x, y)$. All the eigenvalues of $A_{f}(G)$ are real, say $\lambda_{1}, \lambda_{2}, \cdots, \lambda_{n}$ and the spectral radius is $\rho\left(A_{f}(G)\right)=\max _{1 \leq i \leq n}\left|\lambda_{i}\right|$.

Although the study on the graphical function-indices $T I_{f}$ and $H_{f}$, and on the $f$-weighted adjacency matrix $A_{f}$ has started only in recent years, there have been quite a few publications about them. So, after the above preparations, we are going to survey the results that we know in this direction. Though $H_{f}$ can be reduced to a $T I_{f}$, we give separate surveys to them because $H_{f}$ has its own advantages due to the real function $f(x)$ with only a single variable.

\section{Results on the graphical function-index $H_{f}(G)$}

Before proceeding, we need more notation and terminology as given below.

Given a real function $f(x)$, let $f_{1}(x)=f(x+1)-f(x)$ and $f_{11}=\left(f_{1}\right)_{1}$. We say that $f$ is convex (concave) if $f_{11} \geq(\leq) 0$, and $f$ is strictly convex (concave) if $f_{11}>(<) 0$.

Recently, Tomescu in $[43,44]$ studied $H_{f}(G)$ for a convex function $f$. He gave some upper bounds for the function-index $H_{f}(G)$ and the function $f$ is required to satisfy some other properties except for the convexity. These results are stated as follows.

Theorem 3.1. [43] If $G \in G(n, m)$ maximizes (minimizes) $H_{f}(G)$ where $f(x)$ is strictly convex (concave), then $G$ has at most one nontrivial connected component $C$ and $C$ has a vertex of degree $|V(C)|-1$.

Theorem 3.2. [44] Let $n \geq 2$ and $G \in G(n, m)$ such that $1 \leq m \leq n-1$. If $f(x)$ is a strictly convex function having property that $f(x)$ is differentiable and its derivative is strictly convex, then it holds that

$$
H_{f}(G) \leq f(m)+m f(1)+(n-m-1) f(0),
$$

with equality if and only if $G=S_{m+1} \cup(n-m-1) K_{1}$. 
Theorem 3.3. [44] If $n \geq 3, n \leq m \leq 2 n-3, f(x)$ is a strictly convex function having property that $f(x)$ is differentiable and its derivative is strictly convex, and $G \in G(n, m)$ is connected, then it holds that

$$
H_{f}(G) \leq f(n-1)+f(m-n+2)+(m-n+1) f(2)+(2 n-m-3) f(1),
$$

with equality if and only if $G=K_{1} \vee\left(K_{1, m-n+1} \cup(2 n-m-3) K_{1}\right)$ (see Figure 1).

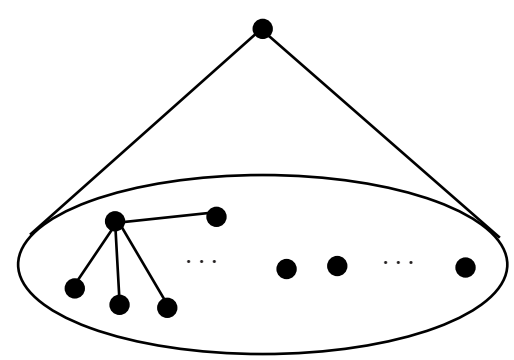

Figure 1: The maximal graph $K_{1} \vee\left(K_{1, m-n+1} \cup(2 n-m-3) K_{1}\right)$ in Theorem 3.3.

As one can see, Tomescu's results are all about the upper bounds of $H_{f}(G)$. Ali et al. in [1] gave the following lower bound for connected $(n, m)$-graphs with fewer edges.

Theorem 3.4. [1] Let $n \geq 4$ and $n+1 \leq m \leq 3 n / 2$. If $f(x)$ is a convex (concave) function, then among all connected $(n, m)$-graphs, the graphs $G$ in $\mathcal{G}(n, m)$ minimize $H_{f}(G)$.

Let $f$ be a strictly convex function. Recently, we proved in [27] that the extremal graphs are exactly the almost regular graphs, which covers the result of Ali et al.

Theorem 3.5. [27] Let $n \geq 2$ and $G$ be an (n,m)-graph with $1 \leq m \leq n(n-1) / 2$, and let $k=\lfloor 2 m / n\rfloor$ and $r=2 m-k n$. If $f$ is a strictly convex function, then it holds that

$$
H_{f}(G) \geq r f(k+1)+(n-r) f(k)
$$

and the equality holds if and only if $G \in \mathcal{G}(n, m)$.

Note that graphs in $\mathcal{G}(n, m)$ can be disconnected (see Figure 2). We then constructed connected graphs to show the following result.
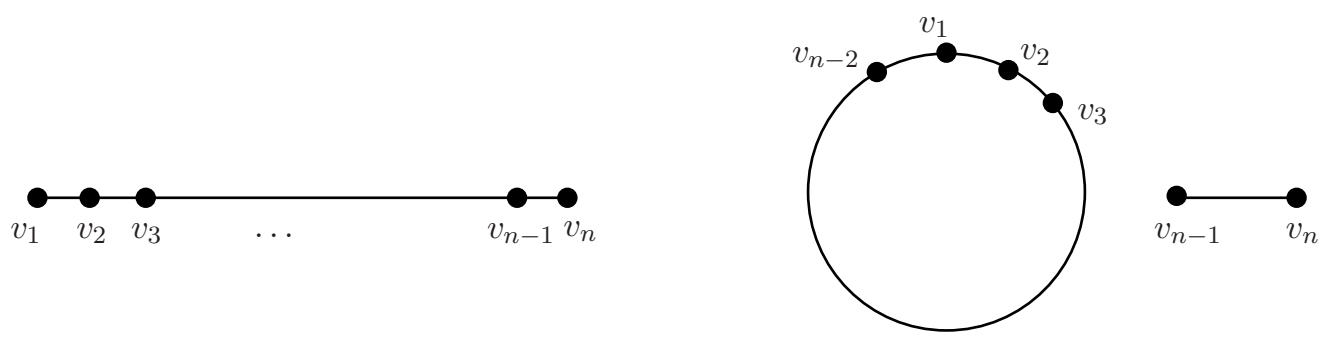

Figure 2: Some connected and disconnected almost regular graphs when $m=n-1$.

Theorem 3.6. [27] Let $n \geq 2$ and $G$ be a connected (n,m)-graph with $n-1 \leq m \leq n(n-1) / 2$, and let $k=\lfloor 2 m / n\rfloor$ and $r=2 m-k n$. If $f$ is a strictly convex function, then it holds that

$$
H_{f}(G) \geq r f(k+1)+(n-r) f(k),
$$

and the equality holds if and only if $G$ is connected and $G \in \mathcal{G}(n, m)$.

Actually, in [27] we also elaborated some algorithms to generate all the minimal graphs and minimal connected graphs, respectively. It is easy to see that $\mathcal{G}(n, m) \neq \emptyset$, that is, there always exists a graph $G$ with degree sequence $\boldsymbol{d}=\left(d_{1}, d_{2}, \ldots, d_{n}\right)$ where $d_{i}=k+1$ and $d_{j}=k$ for $1 \leq i \leq r$ and $r+1 \leq j \leq n$. In fact, it is easy to see that the degree sequence is graphical simply by verifying the conditions in [23]. At first, we give an algorithm to construct some connected graphs with this degree sequence. 


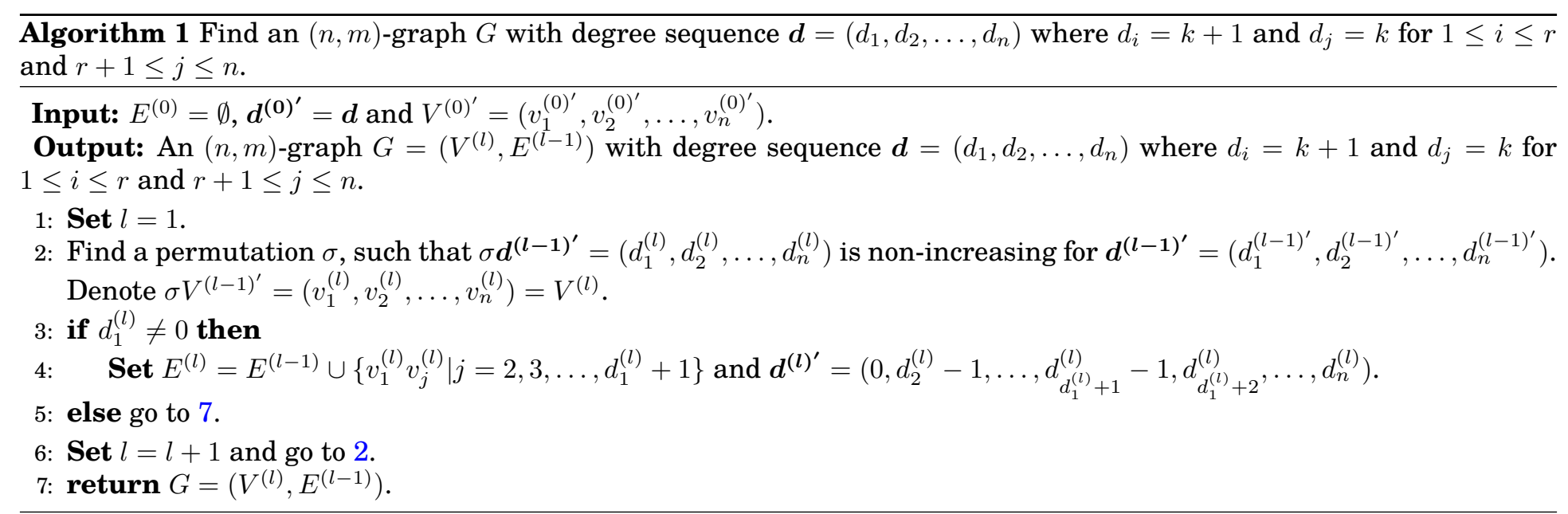

By choosing different permutations $\sigma$ in Algorithm 1, we can obtain some connected $(n, m)$-graphs $G \in \mathcal{G}(n, m)$ which minimize the value of $H_{f}(G)$ for every pairs of $n$ and $m$. However, from [38] we can get the following algorithm, which can generate all graphs of $\mathcal{G}(n, m)$.

Algorithm 2 Find all $(n, m)$-graphs with degree sequence $\boldsymbol{d}=\left(d_{1}, d_{2}, \ldots, d_{n}\right)$ where $d_{i}=k+1$ and $d_{j}=k$ for $1 \leq i \leq r$ and $r+1 \leq j \leq n$.

Input: $n, m$ and $\boldsymbol{d}=\left(d_{1}, d_{2}, \ldots, d_{n}\right)$ where $d_{i}=k+1$ and $d_{j}=k$ for $1 \leq i \leq r$ and $r+1 \leq j \leq n$.

Output: $\mathcal{G}(n, m)$ for any given $n$ and $m$.

1: Construct a complete $n$-partite graph $H=\left(P_{1}, P_{2}, \ldots, P_{n}\right)$, such that each $P_{i}$ for $1 \leq i \leq r$ has $k+1$ vertices and each $P_{j}$ for $r+1 \leq j \leq n$ has $k$ vertices.

2: Find all perfect matchings in $H$, denoted by $\left\{M_{1}, M_{2}, \ldots, M_{l}\right\}$.

3: Set $\mathcal{G}(n, m)=\emptyset$ and $s=1$.

4: while $s \leq l$ do

5: $\quad$ Construct a new graph $G_{s}$ with vertex-set $\left\{p_{1}, p_{2}, \ldots, p_{n}\right\}$ and $p_{i} \sim p_{j}$ if and only if there is an edge between $P_{i}$ and $P_{j}$ in $M_{s}$.

6: $\quad$ if $G_{s}$ does not have multiple edges and $G_{s} \supsetneqq G$ for any $G \in \mathcal{G}(n, m)$ then

7: $\quad$ Set $\mathcal{G}(n, m)=\mathcal{G}(n, m) \bigcup\left\{G_{s}\right\}$.

8: $\quad$ else $\mathcal{G}(n, m)=\mathcal{G}(n, m)$.

9: $\quad$ Set $s=s+1$ and go to 4 .

10: return $\mathcal{G}(n, m)$.

Note that to check that $G_{s} \supsetneqq G$ for any $G \in \mathcal{G}(n, m)$ is a very hard nut to crack. Although this algorithm can be used to generate all graphs of $\mathcal{G}(n, m)$, it cannot guarantee the existence of any graph in $\mathcal{G}(n, m)$.

The following Algorithm 3 (similar to Algorithm 2) can be used to find all connected graphs in $\mathcal{G}(n, m)$.

Algorithm 3 Find all connected $(n, m)$-graphs with degree sequence $\boldsymbol{d}=\left(d_{1}, d_{2}, \ldots, d_{n}\right)$ where $d_{i}=k+1$ and $d_{j}=k$ for $1 \leq i \leq r$ and $r+1 \leq j \leq n$.

Input: $n, m$ and $\boldsymbol{d}=\left(d_{1}, d_{2}, \ldots, d_{n}\right)$ where $d_{i}=k+1$ and $d_{j}=k$ for $1 \leq i \leq r$ and $r+1 \leq j \leq n$.

Output: All connected graphs in $\mathcal{G}(n, m)$ for any given $n$ and $m$, denoted by $\mathcal{G}^{*}(n, m)$.

1: Construct a complete $n$-partite graph $H=\left(P_{1}, P_{2}, \ldots, P_{n}\right)$, such that each $P_{i}$ for $1 \leq i \leq r$ has $k+1$ vertices and each $P_{j}$ for $r+1 \leq j \leq n$ has $k$ vertices.

2: Find all perfect matchings in $H$, denoted by $\left\{M_{1}, M_{2}, \ldots, M_{l}\right\}$.

3: Set $\mathcal{G}^{*}(n, m)=\emptyset$ and $s=1$.

4: while $s \leq l$ do

5: $\quad$ Construct a new graph $G_{s}$ with vertex-set $\left\{p_{1}, p_{2}, \ldots, p_{n}\right\}$ and $p_{i} \sim p_{j}$ if and only if there is an edge between $P_{i}$ and $P_{j}$ in $M_{s}$.

6: $\quad$ if $G_{s}$ is connected with no multiple edges and $G_{s} \nsucceq G$ for any $G \in \mathcal{G}^{*}(n, m)$ then

7: $\quad$ Set $\mathcal{G}^{*}(n, m)=\mathcal{G}^{*}(n, m) \bigcup\left\{G_{s}\right\}$.

8: $\quad$ else $\mathcal{G}^{*}(n, m)=\mathcal{G}^{*}(n, m)$.

9: $\quad$ Set $s=s+1$ and go to 4 .

10: return $\mathcal{G}^{*}(n, m)$. 
Note that although Algorithm 3 can be used to generate all connected graphs of $\mathcal{G}(n, m)$, it cannot guarantee the existence of any connected graph in $\mathcal{G}(n, m)$.

Remark 3.1. As one can see, there are still problems that are left unsolved for the extremal structures on $H_{f}$; for examples, if we consider graphs with more edges, i.e., $m \geq 2 n-2$ edges, what will happen about the maximal graphs in $G(n, m)$ ?

\section{Results on the graphical function index $T I_{f}(G)$}

Rada and Cruz in [39] obtained some extremal results for graphs in $G(n)$ with $n$ vertices but without isolated vertices. For a topological index $T I_{f}$ with an associated function $g(i, j)=\frac{i j f(i, j)}{i+j}$, define the sets

$$
K_{\min }(g)=\{(r, s) \in K: g(r, s)=\min g(i, j)\}
$$

and

$$
K_{\max }(g)=\{(r, s) \in K: g(r, s)=\max g(i, j)\},
$$

and the complements of $K_{\min }(g)$ and $K_{\max }(g)$ in $K$ are denoted by $K_{\min }^{c}(g)$ and $K_{\max }^{c}(g)$, respectively. Their results are stated as follows.

Theorem 4.1. [39] Let $g(i, j)=\frac{i j f(i, j)}{i+j}$. Then for every $G \in G(n)$,

$$
n\left(\min _{(i, j) \in K} g(i, j)\right) \leq T I_{f}(G) \leq n\left(\max _{(i, j) \in K} g(i, j)\right),
$$

Moreover, equality on the left-hand side occurs if and only if $m_{p q}=0$ for all $(p, q) \in K_{\min }^{c}(g)$, while equality on the right-hand side occurs if and only if $m_{r s}=0$ for all $(r, s) \in K_{\max }^{c}(g)$.

Theorem 4.2. [39] Let $g(i, j)=\frac{i j f(i, j)}{i+j}$. Assume $K_{\min }(g)=\{(1,1)\}$ and $n$ is odd. If $g(1,2)<g(i, j)$ for all $(i, j) \in K$ such that $(i, j) \notin\{(1,1),(1,2)\}$, Then for every $G \in G(n)$,

$$
T I_{f}(G) \geq(n-3) g(1,1)+3 g(1,2),
$$

and equality occurs if and only if $G=\frac{n-3}{2} K_{2} \cup P_{3}$ (see Figure 3).

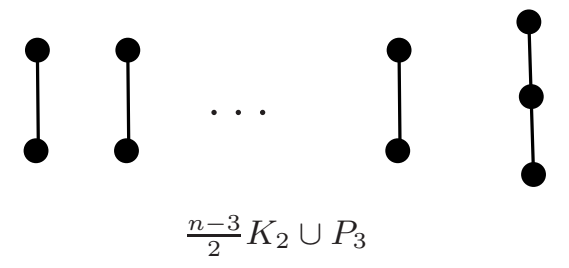

Figure 3: The minimal graph in Theorem 4.2.

Theorem 4.3. [39] Let $g(i, j)=\frac{i j f(i, j)}{i+j}$. Assume that $K_{\max }(g)=\{(1,1)\}$ and $n$ is odd.

1. If $g(1,2) \geq g(2,2) \geq g(i, j)$ for all $(i, j) \in K$ such that $(i, j) \notin\{(1,1),(1,2),(2,2)\}$, then

$$
T I_{f}(G) \leq(n-3) g(1,1)+3 g(1,2)
$$

for all graphs $G \in G(n)$, and equality occurs if and only if $G=\frac{n-3}{2} K_{2} \cup P_{3}$ (see Figure 4).

2. If $g(2,2) \geq g(1,2) \geq g(i, j)$ for all $(i, j) \in K$ such that $(i, j) \notin\{(1,1),(1,2),(2,2)\}$, then

$$
T I_{f}(G) \leq(n-3) g(1,1)+3 g(2,2)
$$

for all graphs $G \in G(n)$, and equality occurs if and only if $G=\frac{n-3}{2} K_{2} \cup C_{3}$ (see Figure 4).

3. If $g(1,2)=g(2,2) \geq g(i, j)$ for all $(i, j) \in K$ such that $(i, j) \notin\{(1,1),(1,2),(2,2)\}$, then

$$
T I_{f}(G) \leq(n-3) g(1,1)+3 g(1,2)=(n-3) g(1,1)+3 g(2,2)
$$

for all graphs $G \in G(n)$, and equality occurs if and only if $G=\frac{n-3}{2} K_{2} \cup P_{3}$ or $\frac{n-3}{2} K_{2} \cup C_{3}$. 


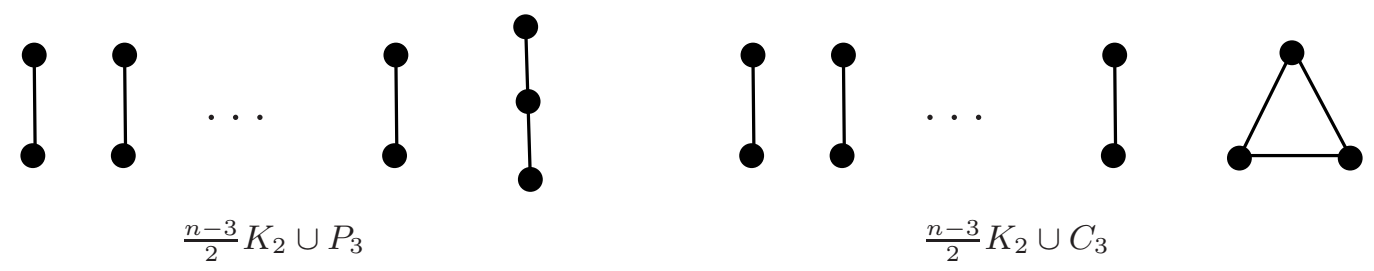

Figure 4: The maximal graphs in Theorem 4.3.

Later, Cruz and Rada in [9] obtained some extremal results for trees with the weight function being an exponential type. Let $\mathcal{T}_{n}$ be the set of all trees on $n$ vertices. It is well known that $P_{n}$ and $S_{n}$ are extremal graphs with respect to most of graphical indices over $\mathcal{T}_{n}$, as we can see in Table 2 from [5, 13, 15, 16, 34, 35, 47, 48, 51,52].

Table 2: Extremal trees for some graphical indices.

\begin{tabular}{ccccccccc}
\hline & $M_{1}$ & $M_{2}$ & $\chi$ & $H$ & $G A$ & $S C$ & $A B C$ & $A Z$ \\
\hline minimal tree & $P_{n}$ & $P_{n}$ & $S_{n}$ & $S_{n}$ & $S_{n}$ & $S_{n}$ & $?$ & $S_{n}$ \\
maximal tree & $S_{n}$ & $S_{n}$ & $P_{n}$ & $P_{n}$ & $P_{n}$ & $P_{n}$ & $S_{n}$ & $S_{\lfloor n / 2-1\rfloor,\lceil n / 2-1\rceil}(n \geq 19)$ \\
\hline
\end{tabular}

The exponential of $T I_{f}$ is defined as the edge-weighted topological index $e^{T I_{f}}$ induced by

$$
e^{T I_{f}}(G)=\sum_{u v \in E(G)} e^{f\left(d_{u}, d_{v}\right)}
$$

The results of exponential topological indices from $[8,9,11,50]$ are summarized in Table 3.

Table 3: Extremal trees for some graphical function-indices with weight-functions being exponential types.

\begin{tabular}{ccccccccc}
\hline & $e^{M_{1}}$ & $e^{M_{2}}$ & $e^{\chi}$ & $e^{H}$ & $e^{G A}$ & $e^{S C}$ & $e^{A B C}$ & $e^{A Z}$ \\
\hline minimal tree & $P_{n}$ & $P_{n}$ & $S_{n}$ & $S_{n}$ & $S_{n}$ & $S_{n}$ & $?$ & $S_{n}$ \\
maximal tree & $S_{n}$ & $S_{\lfloor n / 2-1\rfloor,\lceil n / 2-1\rceil}$ & $P_{n}$ & $P_{n}$ & $P_{n}$ & $P_{n}$ & $S_{n}$ & $S_{\lfloor n / 2-1\rfloor,\lceil n / 2-1\rceil}$ \\
\hline
\end{tabular}

One can see that the above know results are only about graphs on $n$ vertices but without given number $m$ of edges, or about functions $f(x, y)$ of exponential type. Recently, we studied graphs with $n$ vertices and $m$ edges, and with much more general functions $f(x, y)$. Before proceeding to summarizing our results, we need the following notation and terminology about the edge-weight function $f(x, y)$. Let $f_{1}(x, y)=f(x+1, y)-f(x, y)$ and $f_{2}(x, y)=f(x, y+1)-f(x, y)$. Naturally, let $f_{11}:=\left(f_{1}\right)_{1}$ and $f_{12}:=\left(f_{1}\right)_{2} . f(x, y)$ is called (strictly) monotonically increasing if $f_{1}$ is non-negative (no-positive). Notice that if $f(x, y)$ is partial differentiable and $\frac{\partial f}{\partial x}$ is positive (non-negative), then $f_{1}$ is positive (non-negative). The convexity of a real function is stated as follows: $f(x, y)$ is called convex if for any $\left(x_{1}, y_{1}\right),\left(x_{2}, y_{2}\right)$ and $\mu \in(0,1), f\left(\mu x_{1}+(1-\mu) x_{2}, \mu y_{1}+\right.$ $\left.(1-\mu) y_{2}\right) \leq \mu f\left(x_{1}, y_{1}\right)+(1-\mu) f\left(x_{2}, y_{2}\right)$. Notice that the convexity of $f$ implies that $f_{11} \geq 0$. The following properties of a function will frequently appear. We say that a function $f(x, y)$ has the property $P\left(P^{\prime}\right)$ if for any $x_{1}+y_{1}=x_{2}+y_{2}$ and $\left|x_{1}-y_{1}\right|>\left|x_{2}-y_{2}\right|, f\left(x_{1}, y_{1}\right)>(<) f\left(x_{2}, y_{2}\right)$. It is not difficult to see that a symmetric and convex function has the property $P$.

Recently, we studied the extremal problems in [26] for $T I_{f}$ in graph family $G(n, m)$ consisting of graphs with $n$ vertices and $m$ edges, and got the following results.

Theorem 4.4. [26] Let $n$ and $m$ be integers such that $n \geq 2$ and $1 \leq m \leq n(n-1) / 2$. If $f(x, y)$ is convex and partial differentiable with $\frac{\partial f}{\partial x} \geq 0$, then we have

$$
T I_{f}(G) \geq m f(2 m / n, 2 m / n)
$$

for any $(n, m)$-graph $G$, and the bound is sharp since all regular graphs can achieve the lower bound.

For graphs with very few edges, we got the following results.

Theorem 4.5. [26] Let $n$ and $m$ be integers such that $n \geq 2$ and $1 \leq m \leq n / 2$. If $f(x, y)$ is symmetric and $f_{1}>0$, then for any $(n, m)$-graph $G$, we have $T I_{f}(G) \geq m f(1,1)$, and the equality holds if and only if $G=m K_{2} \cup(n-2 m) K_{1}$ (see Figure 5).

Notice that these minimal graphs are all almost regular graphs. Next, for graphs with a little more edges, we have the following result. 


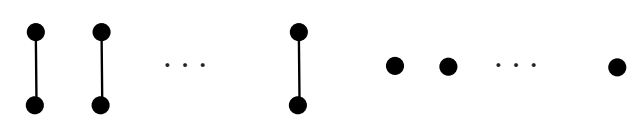

Figure 5: The maximal graph $m K_{2} \cup(n-2 m) K_{1}$ in Theorem 4.5.

Theorem 4.6. [26] Suppose $n$ and $m$ are integers such that $n \geq 2$ and $n / 2 \leq m \leq n-1$. If $f(x, y)$ satisfies that $f_{1}>0$, $f_{11}>0, f_{12} \geq 0$ and $f(1,3)>f(2,2)$, then every minimal graph in $G(n, m)$ is an almost regular graph. Moreover, if $f(1,1)+f(2,2)=2 f(1,2)$, then every almost regular graphs is also a minimal graph in $G(n, m)$.

For a nontrivial component $G_{1}$ of a graph $G$, a vertex $v$ is a universal vertex in $G_{1}$ if $d_{v}=\left|V\left(G_{1}\right)\right|-1$. In this situation, the maximal graphs have the following property.

Theorem 4.7. [26] Suppose $n$ and $m$ are integers such that $n \geq 2$ and $1 \leq m \leq n(n-1) / 2$. If $f(x, y)$ has the property $P$ and satisfies that $f_{1}>0$ and $f_{11}>0$, then the maximal graphs in $G(n, m)$ have exactly one nontrivial component, and the component has a universal vertex.

When $m \leq n-1$, the unique maximal graph is the union of a star and some isolated vertices.

Theorem 4.8. [26] Suppose $n$ and $m$ are integers such that $n \geq 2$ and $1 \leq m \leq n-1$. Let $G$ be an (n,m)-graph. If $f(x, y)$ has the property $P$ and satisfies $f_{1}>0$, then $T I_{f}(G) \leq m f(1, m)$, and the equality holds if and only if $G=K_{1, m} \cup(n-m-1) K_{1}$.

When $m$ is larger, the unique maximal graph among all connected $(n, m)$-graphs with fewer edges is shown as follows.

Theorem 4.9. [26] Suppose $n$ and $m$ are integers such that $n \geq 3$ and $n-1 \leq m \leq 2 n-3$. Let $\gamma=m-n+1$ and $\mathcal{G}^{c}(n, m)$ be the family of connected graphs with $n$ vertices and m edges. If $f(x, y)$ has the property $P$ and satisfies that $f_{1}>0, f_{11}>0$ and $f_{111} \geq 0$, then we have that for any $G \in \mathcal{G}^{c}(n, m), T I_{f}(G) \leq(n-\gamma-2) f(n-1,1)+\gamma f(n-1,2)+\gamma f(\gamma+1,2)+f(n-1, \gamma+1)$, and the equality holds if and only if $G=K_{1} \vee\left(K_{1, \gamma} \cup(n-\gamma-2) K_{1}\right)$ (see Figure 6).

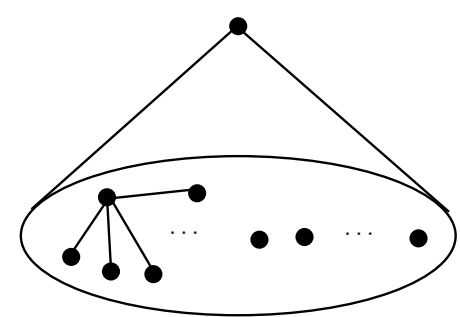

Figure 6: The maximal graph $K_{1} \vee\left(K_{1, \gamma} \cup(n-\gamma-2) K_{1}\right.$ in Theorem 4.9.

Remark 4.1. Similarly as one can see, there are still problems that are left unsolved for the extremal structures on $T I_{f}$, for examples, if we consider graphs with more edges, i.e., $m \geq 2 n-2$ edges, what will happen about the maximal graphs in $G(n, m)$ ?

\section{Extremal spectral radius results on the $f$-weighted adjacency matrix $A_{f}$}

The spectral properties, such as spectral radius, maximum and minimum eigenvalues, energy, etc. should be studied for these $f$-weighted adjacency matrices. Actually, there have already been many publications along with this topic. Here we would like to point out that the $f$-weighted adjacency matrices of graphs with degree-based weights behave quite different from the traditional $(0,1)$-adjacency matrix, for example, there is no monotonicity for the spectral radius, for example, if the weight function $f$ is taken from the $\mathrm{ABC}$-index.

We have obtained the following results on the extremal spectral radius problem.

Theorem 5.1. [10] Let $T$ be a tree of order $n \geq 3$, and $A(T)$ is the adjacency matrix of $T$. Then

$$
\rho\left(A\left(P_{n}\right)\right) \leq \rho(A(T)) \leq \rho\left(A\left(S_{n}\right)\right) .
$$

The equalities hold if and only if $T \cong P_{n}$ or $S_{n}$, respectively. 
Theorem 5.2. [37] Let $f(x, y)=1 / \sqrt{x y}$ be the weight function for Randić index. If $G$ is a non-empty graph, then

$$
\rho\left(A_{f}(G)\right)=1 .
$$

Theorem 5.3. [6] Let $f(x, y)=\sqrt{(x+y-2) /(x y)}$ be the weight function for ABC index. Then

$$
\rho\left(A_{f}\left(P_{n}\right)\right) \leq \rho(A(T)) \leq \rho\left(A_{f}\left(S_{n}\right)\right) .
$$

The equalities hold if and only if $T \cong P_{n}$ or $S_{n}$, respectively.

One can see that the extremal graphs vary quite sensitively depending on the weight functions $f$. Our purpose is to study the extremal spectral radius problem in a unified way. So far we have only got the following results.

Theorem 5.4. [33] Assume that $f(x, y)>0$ is a symmetric real function, increasing and convex in variable $x$. Then the tree of order $n$ with largest spectral radius of $A_{f}(T)$ is $S_{n}$ or a double star $S_{d, n-d}$ for some $d \in\{2, \ldots, n-2\}$ (see Figure 7 ).

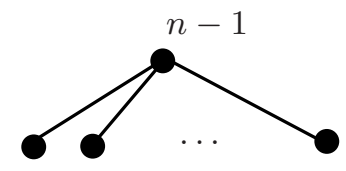

$S_{n}$

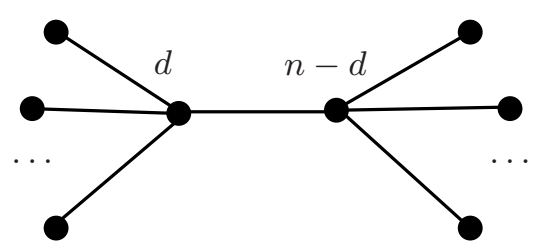

$S_{d, n-d}$

Figure 7: A star and a double star.

It can be seen here that we get new extremal trees i.e., the double stars, which are different from those given in the first three results.

Theorem 5.5. [33] Assume that $f(x, y)$ has a form $P(x, y)$ or $\sqrt{P(x, y)}$, where $P(x, y)$ is a symmetric polynomial with nonnegative coefficients and zero constant term. Then the tree of order $n(n \geq 9)$ with the smallest spectral radius of $A_{f}(T)$ is unique and it is $P_{n}$.

Remark 5.1. Our approach is just a start. More efforts are needed in order to deepen the research on the extremal problems. For examples, one can focus on considering different graph or tree families. One can also consider the weight function $f$ having some other nice properties.

\section{Concluding remarks}

It is just a starting point to unify the solutions for extremal problems of graphical function-indices and $f$-weighted matrix. In this way, we do not need to deal with chemical or graphical indices and degree-based weighted adjacency matrices of graphs with weight functions as various topological indices one by one separately. However, at the moment, we only solved the case when the indices are defined by a symmetric function $f(x, y)$ with some particular properties. For those functions $f(x, y)$ with less restrictions, further study is needed, depending on their functional properties, and on the graph families we focus on. This survey gives a hope that in the near future more results can be worked out for much wider classes of graphical function-indices and $f$-weighted adjacency matrices and/or for much wider families of graphs.

For graph energies, the asymptotic values were obtained in [30-32] for $f$-weighted adjacency and Laplacian matrices with degree based and degree-distance-based entries where the function $f$ has very relaxed conditions; for example, continuous and/or differentiable. Since they are about extremal results, we omit their details in this survey.

\section{Acknowledgment}

This work is supported by the National Natural Science Foundation of China (through grant No.12131013 and 11871034).

\section{References}

[1] A. Ali, I. Gutman, H. Saber, A. M. Alanazi, On bond incident degree indices of ( $n, m)$-graphs, MATCH Commun. Math. Comput. Chem. 87 (2022) $89-96$. 
[2] A. T. Balaban, I. Motoc, D. Bonchev, O. Mekenyan, Topological indices for structure-activity correlations, In: V. Austel, A. T. Balaban, D. Bonchev, M. Charton, T. Fujita, H. Iwamura, O. Mekenyan, I. Motoc (Eds.), Steric Effects in Drug Design, Springer, Berlin, 1983, pp. 21-55.

[3] B. Bollobás, P. Erdös, A. Sarkar, Extremal graphs for weights, Discrete Math. 200 (1999) 5-19.

[4] J. A. Bondy, U. S. R. Murty, Graph Theory, Springer, Berlin, 2008.

[5] G. Caporossi, I. Gutman, P. Hansen, L. Pavlović, Graphs with maximum connectivity index Comput. Biol. Chem. 27 (2003) 85-90.

[6] X. Chen, On extremality of ABC spectral radius of a tree, Linear Algebra Appl. 564 (2019) 159-169

[7] S. M. Cioaba, Sums of powers of the degrees of a graph, Discrete Math. 306 (2008) 959-1964.

[8] R. Cruz, J. Monsalve, J. Rada, Trees with maximum exponential Randić index, Discrete Appl. Math. 283 (2020) 634-643.

[9] R. Cruz, J. Rada, The path and the star as extremal values of vertex-degree-based topological indices among trees, MATCH Commun. Math. Comput. Chem. 82 (2019) 715-732.

[10] D. Cvetković, P. Rowlinson, S. Simić, An Introduction to the Theory of Graph Spectra, Cambridge University Press, New York, 2010.

[11] K. C. Das, S. Elumalai, S. Balachandran, Open problems on the exponential vertex-degree-based topological indices of graphs, Discrete Appl. Math. 293 (2021) 38-49.

[12] K. Das, I. Gutman, I. Milovanović, E. Milovanović, B. Furtula, Degree-based energies of graphs, Linear Algebra Appl. 554 (2018) 185-204.

[13] H. Deng, A unified approach to the extremal Zagreb indices for trees, unicyclic graphs and bicyclic graphs, MATCH Commun. Math. Comput. Chem. 57 (2007) 597-616.

[14] E. Estrada, The ABC matrix, J. Math. Chem. 55 (2017) 1021-1033.

[15] B. Furtula, A. Graovac, D. Vukičević, Atom-bond connectivity index of trees, Discrete Appl. Math. 157 (2009) $2828-2835$.

[16] B. Furtula, A. Graovac, D. Vukičević, Augmented Zagreb index, J. Math. Chem. 48 (2010) 370-380.

[17] B. Furtula, I. Gutman, A forgotten topological index, J. Math. Chem. 53(4) (2015) 1184-1190.

[18] B. Furtula, I. Gutman, M. Dehmer, On structure-sensitivity of degree-based topological indices, Appl. Math. Comput. 219 (2013) $8973-8978$.

[19] I. Gutman, Degree-based topological indices, Croat. Chem. Acta 86 (2013) 351-361.

[20] I. Gutman, Geometric approach to degree-based topological indices: Sombor indices, MATCH Commun. Math. Comput. Chem. 86 (2021) 11-16.

[21] I. Gutman, B. Furtula, Novel Molecular Structure Descriptors - Theory and Applications, Volumes I and II, University of Kragujevac, Kragujevac, Serbia, 2010.

[22] I. Gutman, N. Trinajstić, Graph theory and molecular orbitals. Total $\pi$-electron energy of alternant hydrocarbons, Chem. Phys. Lett. 17 (1972) $535-538$.

[23] V. Havel, A remark on the existence of finite graphs, Casopis Pest. Mat. 6 (1995) 161-179.

[24] Y. Hu, Y. Shi, X. Li, T. Xu, On molecular graphs with smallest and greatest zeroth-order general Randić index, MATCH Commun. Math. Comput. Chem. 54 (2005) 425-434.

[25] Y. Hu, Y. Shi, X. Li, T. Xu, Connected graphs with minimum and maximum zeroth-order general Randić index, Discrete Appl. Math. 155 (2007) $1044-1054$.

[26] Z. Hu, L. Li, X. Li, D. Peng, Extremal graphs for topological index defined by a degree-based edge-weight function, MATCH Commun. Math. Comput. Chem., In press.

[27] Z. Hu, X. Li, D. Peng, Graphs with minimum vertex-degree function-index for convex functions, MATCH Commun. Math. Comput. Chem., In press.

[28] R. Lang, X. Li, S. Zhang, Inverse problem for the Zagreb index of molecular graphs, Appl. Math. J. Chinese Univ. Ser. A 4 (2003) 487-493.

[29] X. Li, I. Gutman, Mathematical Aspects of Randić-Type Molecular Structure Descriptors, University of Kragujevac, Kragujevac, Serbia, 2006.

[30] X. Li, Y. Li, J. Song, The asymptotic value of graph energy for random graphs with degree-based weights, Discrete Appl. Math. 284 (2020) $481-488$.

[31] X. Li, Y. Li, Z. Wang, The asymptotic value of energy for matrices with degree-distance-based entries of random graphs, Linear Algebra Appl. 603 (2020) 390-401.

[32] X. Li, Y. Li, Z. Wang, Asymptotic values of four Laplacian-type energies for matrices with degree-distance-based entries of random graphs, Linear Algebra Appl. 612 (2021) 318-333.

[33] X. Li, Z. Wang, Trees with extremal spectral radius of weighted adjacency matrices among trees weighted by degree-based indices, Linear Algebra Appl. 612 (2021) 61-75.

[34] X. Li, J. Zheng, A unified approach to the extremal trees for different indices, MATCH Commun. Math. Comput. Chem. 54 (2005) 195-208.

[35] W. Lin, D. Dimitrov, R. Škrekovski, Complete characterization of trees with maximal augmented Zagreb index, MATCH Commun. Math. Comput. Chem. 83 (2020) 167-178.

[36] N. Linial, E. Rozenman, An extremal problem on degree sequences of graphs, Graphs Combin. 18 (2002) 573-582.

[37] B. Liu, Y. Huang, J. Feng, A note on the Randić spectral radius, MATCH Commun. Math. Comput. Chem. 68 (2012) 913-916.

[38] M. Molloy, B. Reed, A critical point for random graphs with a given degree sequence, Random Struct. Algorithm. 80 (1955) $477-480$.

[39] J. Rada, R. Cruz, Vertex-degree-based topological indices over graphs, MATCH Commun. Math. Comput. Chem. 72(3) (2014) 603-616.

[40] M. Randić, Characterization of molecular branching, J. Amer. Chem. Soc. 97(23) (1975) 6609-6615.

[41] G. Su, M. Meng, L. Cui, Z. Chen, X. Lan, The general zeroth-order Randić index of maximal outerplanar graphs and trees with $k$ maximum degree vertices, Sci. Asia 43 (2017) 387-393.

[42] R. Todeschini, V. Consonni, Molecular Descriptors for Chemoinformatics, Wiley-VCH, Weinheim, 2009.

[43] I. Tomescu, Properties of connected $(n, m)$-graphs extremal relatively to vertex degree function index for convex functions, MATCH Commun. Math. Comput. Chem. 85 (2021) 285-294.

[44] I. Tomescu, Graphs with given cyclomatic number extremal relatively to vertex degree function index for convex functions, MATCH Commun. Math. Comput. Chem. 87 (2022) 109-114.

[45] N. Trinajstić, Chemical Graph Theory, Second Edition, CRC Press, Boca Raton, 1992.

[46] D. Vukičević, J. Đurđević, Bond additive modeling 10. Upper and lower bounds of bond incident degree indices of catacondensed fluoranthenes, Chem. Phys. Lett. 515 (2011) 186-189.

[47] D. Vukičević, B. Furtula, Topological index based on the ratios of geometrical and arithmetical means of end-vertex degrees of edges, J. Math. Chem. 46 (2009) 1369-1376.

[48] X. Xiao, X. Qiu, W. Lin, Erratum to "complete characterization of trees with maximal augmented Zagreb index", MATCH Commun. Math. Comput. Chem. 87 (2022) 171-176.

[49] Y. Yao, M. Liu, F. Belardo, C. Yang, Unified extremal results of topological indices and spectral invariants of graphs, Discrete Appl. Math. 271 (2019) 218-232.

[50] M. Zeng, H. Deng, An open problem on the exponential of the second Zagreb index, MATCH Commun. Math. Comput. Chem. 85 (2021) 367-373.

[51] L. Zhong, The harmonic index for graphs, Appl. Math. Lett. 25 (2012) 561-566.

[52] B. Zhou, N. Trinajstić, On a novel connectivity index, J. Math. Chem. 46 (2009) 1252-1270. 